\title{
Three-Dimensional Classification of Thoracic Scoliotic Curves
}

\author{
Archana P. Sangole, PhD, ${ }^{*} \dagger$ Carl-Eric Aubin, PhD, ${ }^{*} \dagger$ Hubert Labelle, MD, $\dagger$ \\ Ian A. F. Stokes, PhD, $\neq$ Lawrence G. Lenke, MD, $\mathbb{S}$ Roger Jackson, MD, \\ and Peter Newton, MD\|
}

Study Design. Three-dimensional (3D) characterization of the thoracic scoliotic spine (cross-sectional study).

Objectives. To investigate the presence of subgroups within Lenke type-1 curves by evaluating the thoracic segment indices extracted from $3 \mathrm{D}$ reconstructions of the spine, and to propose a new clinically relevant means (the daVinci representation) to report 3D spinal deformities.

Summary of Background Data. Although scoliosis is recognized to be a 3D deformity of the spine its measurement and classification have predominantly been based on radiographs which are $2 \mathrm{D}$ projections in the coronal and sagittal planes.

Methods. Thoracic segment indices derived from 3D reconstructions of coronal and sagittal standing radiographs of 172 patients with right thoracic adolescent idiopathic scoliosis, reviewed by the 3D Classification Committee of the Scoliosis Research Society, were analyzed using the ISOData unsupervised clustering algorithm. Four curve indices were analyzed: Cobb angle, axial rotation of the apical vertebrae, orientation of the plane of maximum curvature of the main thoracic curve, and kyphosis (T4-T12). No assumptions were made regarding grouping tendencies in the data nor were the number of clusters predefined.

Results. Three primary groups were revealed wherein kyphosis and the orientation of the PMC of the main thoracic curve were the major discriminating factors with slight overlap between groups. A small group (G1) of 22 patients having smaller, nonsurgical (minor) curves was identified. Although the remaining patients had similar Cobb angles they were split into 2 groups (G2: 79 patients; G3: 71 patients) with different PMC (G2: $65^{\circ}-81^{\circ}$; G3: $76^{\circ}-$ $\left.104^{\circ}\right)$ and kyphotic measures (G2: $23^{\circ}-43^{\circ}$; G3: $7^{\circ}-25^{\circ}$ ).

From the *Department of Mechanical Engineering, Ecole Polytechnique de Montréala, Quebec, Canada; †Sainte-Justine University Hospital Centre, Quebec, Canada; $\ddagger$ Department of Orthopedics and Rehabilitation, University of Vermont, Burlington, VT; $\mathbb{D}$ Department of Orthopedic Surgery, WA University, St. Louis, MO; IDepartment of Spine Surgery, North Kansas City Hospital, North Kansas City, MO; and ||Department of Orthopedics, Rady Children's Hospital, San Diego, CA.

Acknowledgment date: May 16, 2008. Acceptance date: May 26, 2008.

The manuscript submitted does not contain information about medical device(s)/drug(s).

Federal (Natural Sciences and Engineering Research Council of Canada) and Other (Scoliosis Research Society) funds were received in support of this work. No benefits in any form have been or will be received from a commercial party related directly or indirectly to the subject of this manuscript.

Supported by grants from Federal (Natural Sciences and Engineering Research Council of Canada) and other (Scoliosis Research Society) funds received for this work.

Address correspondence and reprint requests to Archana P. Sangole, PhD, PEng, École Polytechnique, Department of Mechanical Engineering, P.O. Box 6079, Station Centre-ville, Montreal (Quebec), H3C 3A7, Canada; E-mail: archana.sangole@polymtl.ca
Conclusion. Two distinct subgroups within the surgical cases (major curves) of Lenke type-1 curves were found thus suggesting that thoracic curves are not always hypokyphotic. The ISOData cluster analysis technique helped to capture inherent 3D structural curve complexities that were not evident in a $2 \mathrm{D}$ radiographic plane. The daVinci representation is a new clinically relevant means to report 3D spinal deformities.

Key words: cluster analysis, adolescent idiopathic scoliosis, classification, pattern recognition, daVinci representation. Spine 2009;34:91-99

Scoliosis is recognized to be a three-dimensional (3D) deformity of the spine. It has components not only in the coronal plane but also in the sagittal and transverse planes. ${ }^{1,2}$ Yet, its measurement and classification have predominantly been based on radiographic assessments which are two-dimensional (2D) projections of the spine in the coronal and sagittal planes. Evaluating these projections requires a sense of abstraction and visualization to generate a $3 \mathrm{D}$ perspective not only of the spine curvature that is going to be treated but also of the curvature that could possibly result from a particular surgical instrumentation strategy. The inability to describe the third dimension of scoliosis makes the task all the more difficult. Hence the efforts of the Scoliosis Research Society (SRS) 3D Classification Working Group (1994) towards developing more descriptive indices of the scoliotic spine helped to better characterize the spinal deformity and subsequently describe the third dimension of scoliosis. Recent advances in technology now allow us to have access to more sophisticated algorithms and computing techniques that can be used to facilitate our understanding of scoliosis as a $3 \mathrm{D}$ deformity and thus create more clinically relevant representations of the deformity. ${ }^{3-5}$

The initial attempts at classification of adolescent idiopathic scoliosis (AIS) began in the 1950s, one being Ponseti and Friedman. ${ }^{6}$ Classification of thoracic AIS was first presented in the classic article by King et $a l^{7}$ wherein 5 types of curves were described. Based on this classification they proposed recommendations for selecting fusion levels in patients with thoracic idiopathic scoliosis. Stokes and Aronsson ${ }^{8,9}$ reported better reliability in the King classification if a computer-assisted rulebased algorithm is used as opposed to a subjective evaluation of radiographic features.

Since the King classification, several others have followed and proposed ways to classify scoliotic curves in a 
clinically meaningful manner. A few of these were still limited by the fact that only coronal radiographs were taken into consideration. ${ }^{10}$ The Lenke classification system that uses measurements in both coronal and sagittal planes was introduced in 1997, and has reasonably good reproducibility rating ${ }^{11-15}$ as compared with the King classification system. ${ }^{16}$ It is now used extensively and continues to be further developed with the addition of new indices such as the deformity score or the LenkeHarms score. ${ }^{17}$ Although the King classification primarily focused on thoracic curves, the Lenke classification approaches the issue in a more global sense by proposing a generic classification scheme that also considers the thoracolumbar/lumbar (TL/L) curves.

In an attempt to improve on visual pattern recognition by employing automated classification, Duong et al ${ }^{18}$ proposed an unsupervised clustering technique called the Fuzzy k-means technique to classify the spine curves using shape descriptors derived from 3D reconstruction models of the spine. They identified 5 basic classes of spine curve patterns, which are also present in the King and Lenke classifications and the respective subtypes accordingly divided to reflect clinically identifiable changes in the sagittal plane. The classification method however, is quite complex because it requires knowledge about signal processing techniques and involves the computation of additional parameters that are not intuitive enough to be easily interpreted from the current spine curvature evaluation procedures.

Finding a clinically relevant classification scheme that properly reflects the 3D nature of scoliosis deformity requires defining better indices to characterize the third dimension of scoliosis. Examples such as the torsional component ${ }^{19}$ and axial rotation (Stokes) ${ }^{20}$ have not yet been used in the clinics because of the need for additional measurement tools and the complexity in interpretation. Despite all these efforts to employ classifications of spinal shape and deformity to standardize surgical decisions, there remains substantial variability in surgical planning, primarily due to the surgeon-specific surgical instrumentation preferences. ${ }^{21}$ These efforts should however, be directed towards defining a classification system that perceives scoliosis as a 3D spinal deformity and that which relies on indices of curve patterns derived from coronal and sagittal radiographs so that it is intuitive to the surgeons.

The success of a classification scheme largely depends on the characterization of the deformity and thus the indices used to describe it. The current methods are primarily based on indices defined in 2D planes. The problem is not so much with the indexes used but rather the fundamental nature of the classification schemes i.e., the selective use of an index by projecting the spine onto a single plane and using that as a basis for classification. If the same indices are considered collectively, then together, they represent a 3D characterization of the deformity and the classification scheme thus defined will now be based on the 3D definition of the deformity.
In view of the findings from existing studies, the SRS recognizes the need for $3 \mathrm{D}$ classification and mandates the $3 \mathrm{D}$ classification working group to continue their efforts towards developing a 3D scheme for characterizing scoliosis. This study is part of the same effort and the article presents some of the recent work completed by the committee. The objectives are to analyze the presence of subgroups within Lenke type- 1 curves by evaluating the thoracic segment indices extracted from 3D reconstructions of the spine, and to propose a new clinically relevant means (the daVinci representation) to report 3D spinal deformities.

\section{Materials and Methods}

\section{Patients and Spine Indices}

One hundred seventy-two patients with right thoracic AIS classified as Lenke type 1 by members of the 3D Classification Committee of the SRS were studied. The average age at the time of the visit was $15 \pm 2$ years and the mean thoracic Cobb angle was $43 \pm 14^{\circ}$ (range, $10^{\circ}-76^{\circ}$ ). For each patient, a 3D reconstruction of the spine was obtained from calibrated biplanar radiographs. ${ }^{3}$ The reconstructed models were then used to compute 4 indices of the thoracic segment: the Cobb angle, thoracic kyphosis (measured from T4-T12), axial rotation of the apical vertebra, and the orientation of the plane of maximum curvature with respect to the sagittal plane. ${ }^{22,2}$ Measurements of coronal and sagittal plane curvature were based on a curve fitted mathematically through the centers of the vertebrae. For the scoliosis measurement, inflection points of this line were identified in the coronal plane, and the angle between perpendicular lines at these points provided a measure of scoliosis analogous to the Cobb angle. ${ }^{23}$ For the kyphosis measure

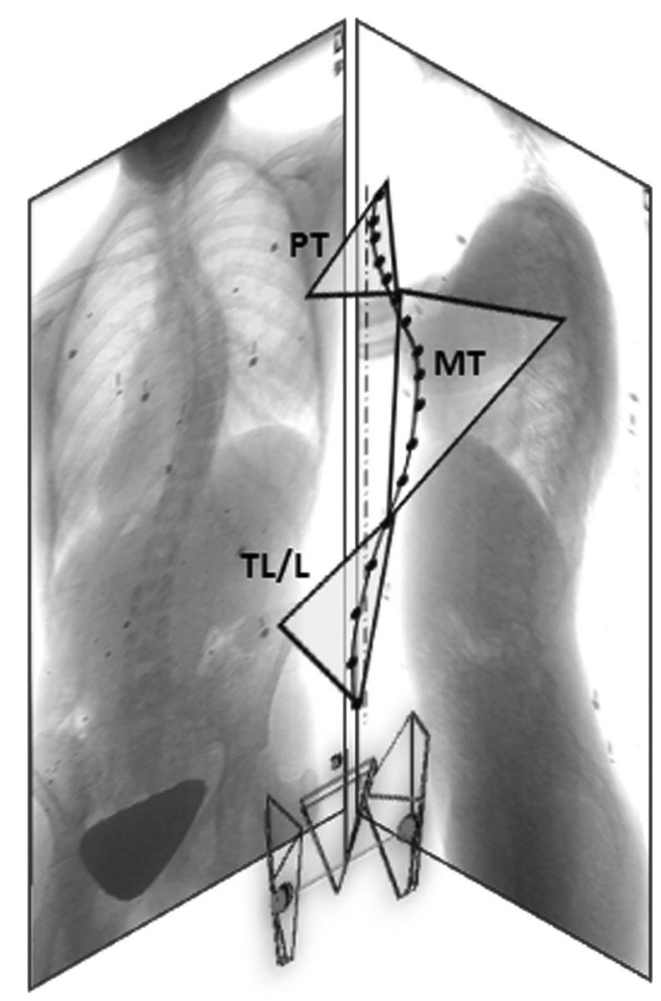

Figure 1. Planes of maximum curvature (PMC) for the proximal thoracic, main thoracic, and lumbar segments. 


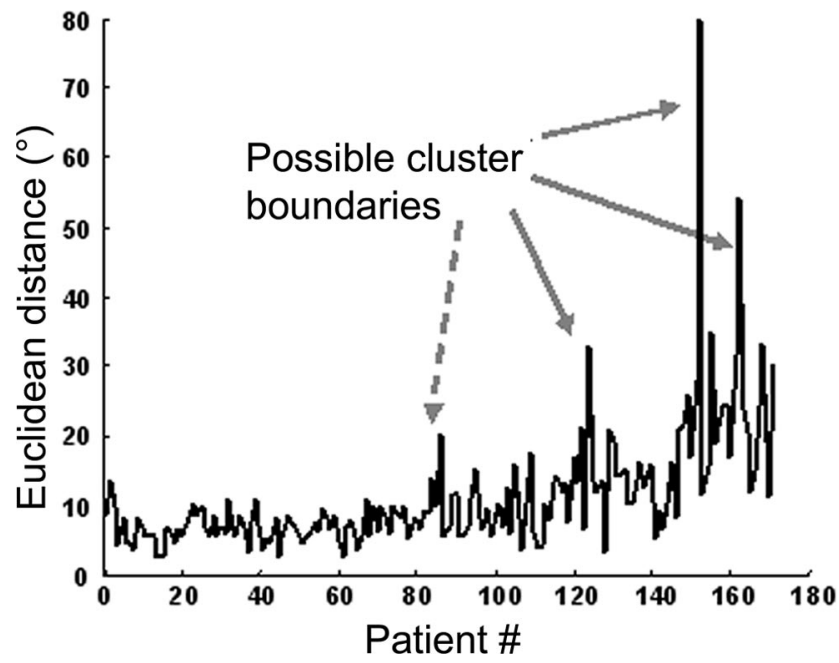

Figure 2. Chainmap of the signature vectors of the 172 patients. The solid arrows indicate the presence of distinct cluster boundaries (prominent spikes with respect to the rest of the plot) while the dotted arrow indicates a possible cluster boundary (relatively short spike).

in the sagittal plane, the angle between tangents at the positions of T4 and T12 were used. Axial rotation of the apical vertebrae was measured about the $\mathrm{z}$-axis in the local XY-plane. ${ }^{24}$ For each segment of the spine, the plane of maximum curvature (PMC) was constructed using the 2 end vertebrae and the apex and their orientation was computed with respect to the sagittal plane (see Figure 1). These 4 indices, when considered collectively represent a "signature" of the scoliotic spine. The 4 pa- rameters can be considered as defining the shape (vector) of that patient's spine in $3 \mathrm{D}$ space.

\section{Three-Dimensional Classification}

An automatic algorithm was used in the analysis to identify clusters of groupings of patients. We used the classical unsupervised clustering method called the Iterative Self-organizing analysis technique of DATA analysis (ISOData) originally introduced by Ball and Hall. ${ }^{25}$ The technique allows the splitting and merging of clusters while trying to find the "best" configuration. It therefore, requires very little a priori knowledge about the data and predefining the possible number of clusters or groups is not required. The range of possible minimum and maximum number of clusters within which the algorithm can search for an optimal configuration was determined by a procedure called the chainmap which reorders the multidimensional data in the form of a $2 \mathrm{D}$ distance map. ${ }^{26}$ A patientvector is randomly selected and its nearest neighbor is identified as that with the shortest distance between the 2 patients' vectors. The closest neighbor to the newly identified vector is likewise identified from the remaining data. This process is continued until the nearest neighbor for each patient-vector is found and the respective distances between nearest neighbors are recorded. The resulting plot is a display of spikes, each spike corresponding to a possible cluster boundary. The prominence of these spikes indicates the distinctiveness of the embedded clusters and thus gives an estimate of the possible number of clusters present in the data. The best configuration identified by splitting and merging clusters, is measured by a quality ratio $(\sigma)$ which is the ratio of the average intercluster variance to the average intracluster variance. The essence is to find a configu-
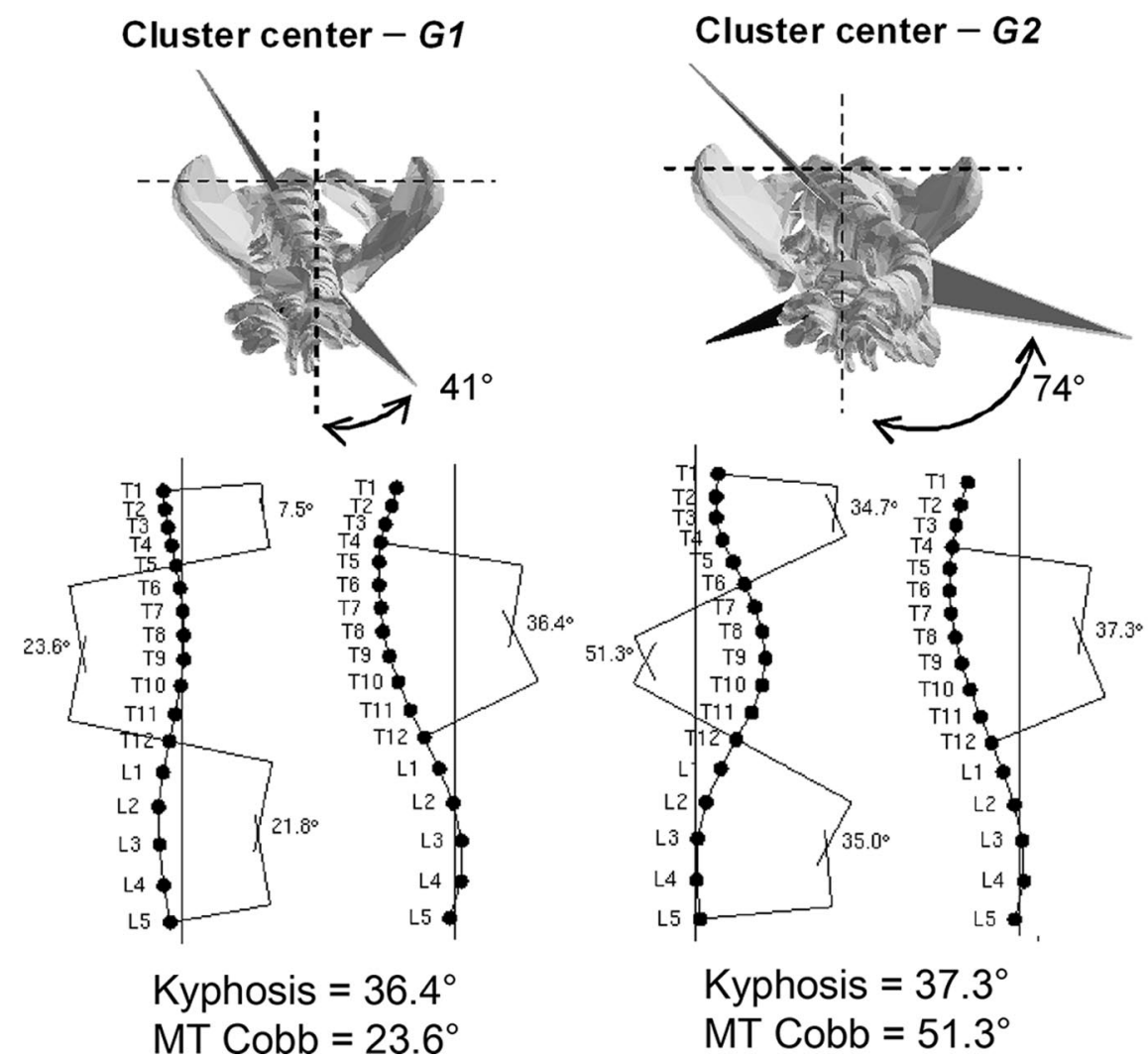

\section{Cluster center - G3}
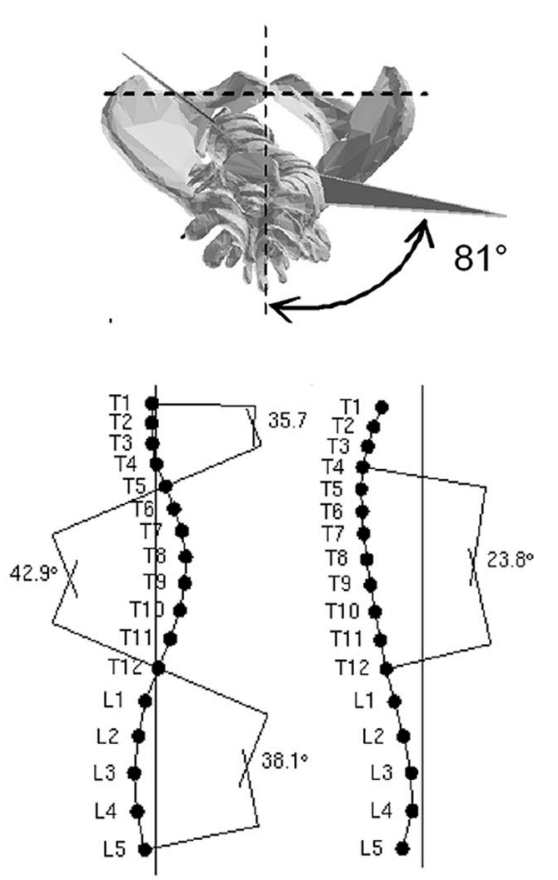

Kyphosis $=23.8^{\circ}$

$\mathrm{MT}$ Cobb $=42.9^{\circ}$

Figure 3. Coronal and sagittal views of the spines of 3 patients who were identified as close to the centers of the 3 clusters G1, G2, and G3. 
Table 1. Average and Standard Deviation Values of the Thoracic Indices Within Each Group

\begin{tabular}{lccccc}
\hline Cluster & Size & $\begin{array}{c}\text { MT Cobb } \\
\text { Angle }\left({ }^{\circ}\right)\end{array}$ & $\begin{array}{c}\text { MT Apex Axial } \\
\text { Rotation }\left({ }^{\circ}\right)\end{array}$ & $\begin{array}{c}\text { MT PMC } \\
\text { Rotation }\left({ }^{\circ}\right)\end{array}$ & $\begin{array}{c}\text { Thoracic } \\
\text { Kyphosis }\left({ }^{\circ}\right)\end{array}$ \\
\hline G1 & 22 & $22.4 \pm 10$ & $-5.7 \pm 6$ & $38.2 \pm 28$ & $35.9 \pm 8$ \\
G2 & 79 & $51.3 \pm 10$ & $-14.8 \pm 9$ & $73.3 \pm 8$ & $33.0 \pm 10$ \\
G3 & 71 & $40.7 \pm 14$ & $-10.9 \pm 8$ & $90.2 \pm 14$ & $16.8 \pm 9$ \\
\hline
\end{tabular}

ration that gives maximal separation between clusters and minimal variance within clusters.

$$
\sigma=\frac{\sum \text { average }(\text { inter }- \text { cluster variance })}{\sum \text { average }(\text { intra }- \text { cluster variance })}
$$

Clusters are merged if (1) there are very few samples assigned to it, (2) if the number of clusters exceeds the maximal range, or (3) if the clusters are too close to each other. Clusters are split if (1) the size of a cluster is too large, or (2) if the number of clusters falls below the minimal range. Only criterion 2 was used in merging and splitting clusters because this reduced the need for a priori assumptions about the data. Additional details about the methods can be found in Ball and Hall, Levinson et al and Memarsadeghi et al. ${ }^{25-27}$

The ISOData clustering algorithm was applied to only main thoracic curves and grouping tendency was evaluated by examining the indices of representative cases from each group. The objective was to examine the presence of subtypes within the Lenke-1 curves. The default initial number of clusters, in all the tests, was set at 10 . No a priori assumptions were made concerning underlying grouping tendencies or the predefined number of groups. A 1-way ANOVA was done to examine the influence of the various parameters on the grouping tendency.

\section{Results}

A chainmap of the thoracic indices shows 3 prominent spikes relative to the rest of the plot thus indicating the presence of at least 3 clusters and a possible fourth cluster indicated by a shorter spike (Figure 2). The ISOData cluster analysis revealed a small group of patients having smaller, nonsurgical (minor) curves ( $G 1=22$ cases) and 2 groups of surgical (major curves) cases with distinct kyphosis and plane of maximum curvature parameters (G2 = 79 cases, $G 3=71$ cases). Coronal and sagittal spine profiles of the representative cases that were identified as the cluster centers are illustrated in Figure 3. The figure shows the coronal and sagittal profiles of the patients and the respective kyphosis, MT Cobb angle and the thoracic plane of maximum curvature orientation. The average values of the thoracic indices within each cluster differed (Table 1) and indicated the presence of a nonsurgical group (G1) and 2 surgical groups (G2 and G3).

Although the 150 surgical cases had almost similar Cobb angles, they were split into 2 groups having almost distinct PMC orientations $\left(G 2: 65^{\circ}-81^{\circ} ; G 3: 76^{\circ}-104^{\circ}\right)$ and kyphosis $\left(G 2: 23^{\circ}-43^{\circ} ; G 3: 7^{\circ}-25^{\circ}\right)$. Representative cases from the surgical groups that have similar MT Cobb angles but different kyphosis measures, as is evident in the radiographs (coronal and sagittal) and trans-
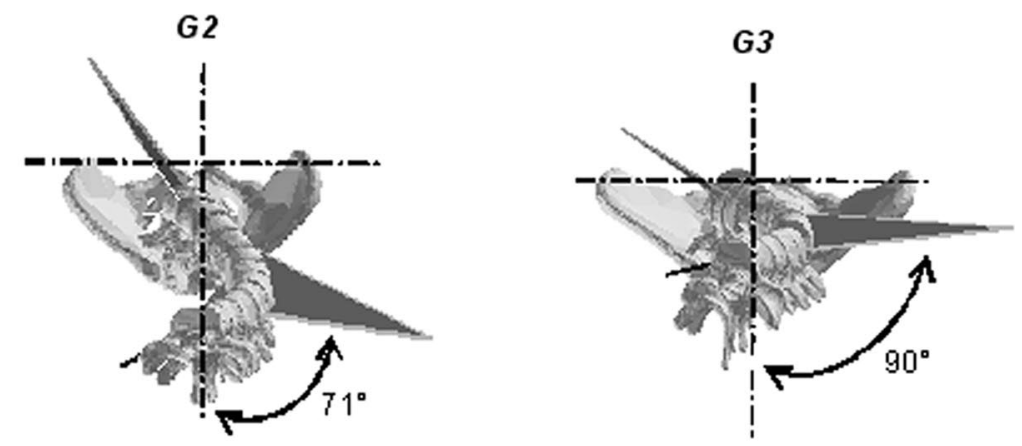

Figure 4. Coronal, sagittal, and top views of representative cases from the 2 surgical (major curves) groups $G 2$ and G3. The 2 patients have similar MT Cobb angles but different kyphosis measures. The planes of maximum curvature are rotated respectively $71^{\circ}$ and $90^{\circ}$ from the sagittal plane.
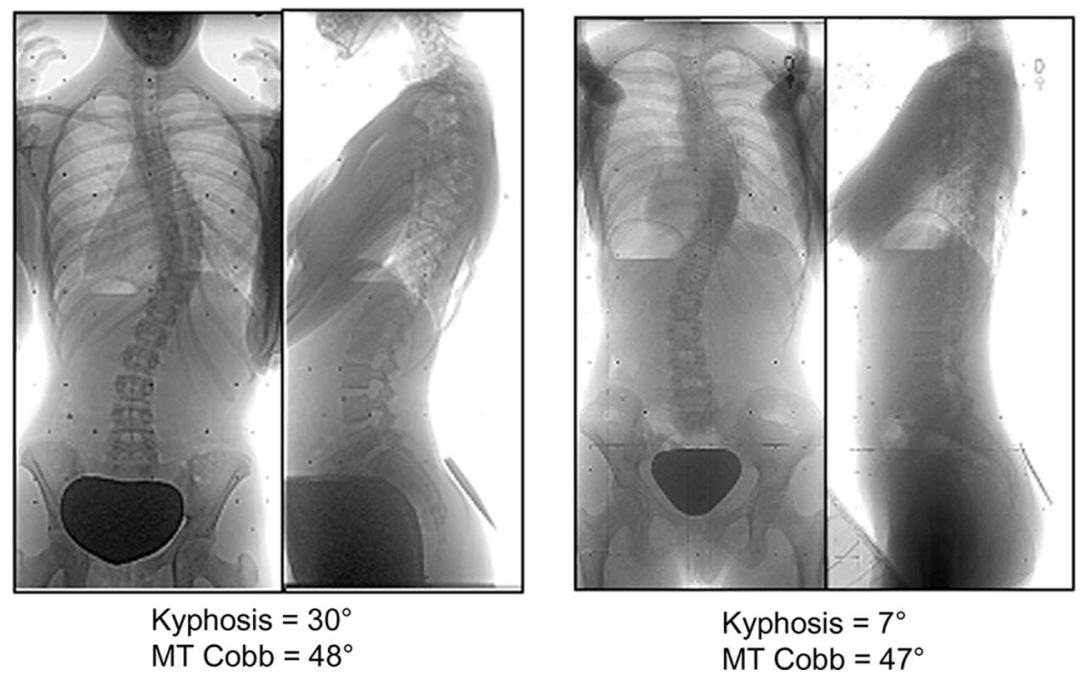

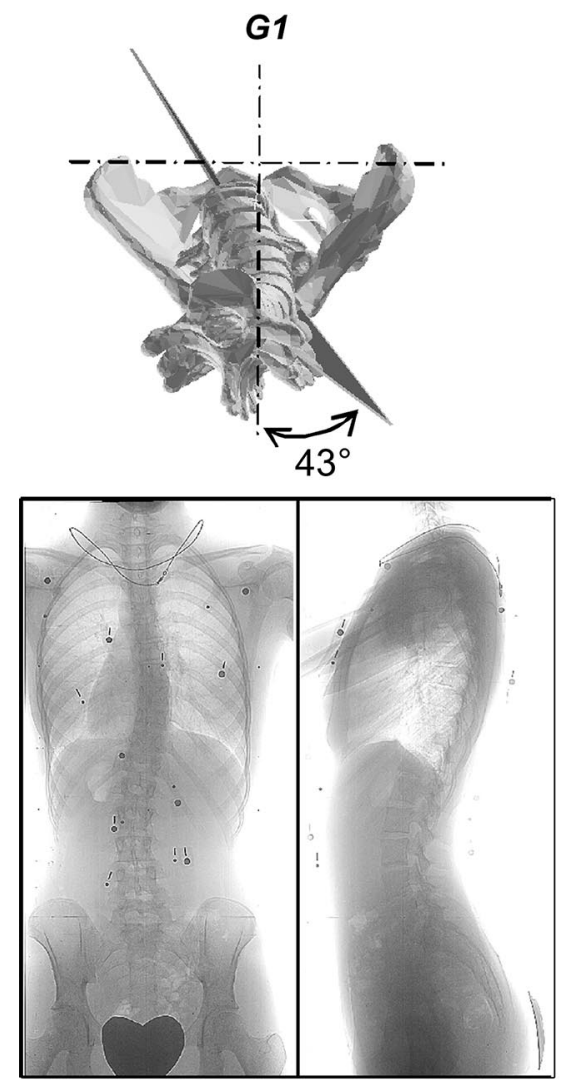

Kyphosis $=34^{\circ}$

$\mathrm{MT}$ Cobb $=21^{\circ}$
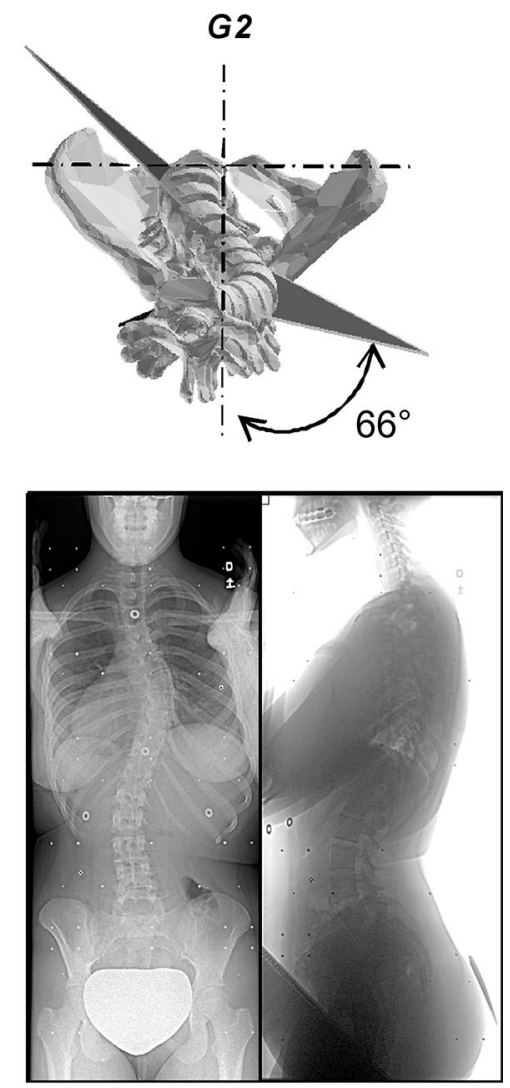

Kyphosis $=33^{\circ}$ $\mathrm{MT}$ Cobb $=44^{\circ}$ second is a surgical case in G2.

verse plane projections of the spine, are presented in Figure 4. There were also cases with similar kyphosis measures but different MT Cobb angles (representative cases shown in Figure 5).

One-way ANOVA analysis showed that all indices, except axial rotation of the apical vertebra, significantly influenced the grouping tendency $(P<0.01)$. The intergroup interactions showed a significant influence of all indices except for the kyphosis measure which was nonsignificant in $G 1$ and $G 2(P>0.5)$ as was expected because $G 1$ and $G 2$ have similar kyphosis measures, but were identified as nonsurgical (minor curves) and surgical (major curves) groups, respectively due to the difference in the MT Cobb and plane of maximum curvature measures.

\section{- Discussion}

In an homogeneous group of surgical thoracic curves as seen in the coronal plane, PMC and the kyphosis measures were the influential parameters that split the cases into 2 subgroups in a 3D analysis. Although the coronal plane profiles of the 2 cases are almost similar there is a remarkable difference in the sagittal profiles, as evident in Figure 4. Since the 2 cases have the same MT Cobb angle intuitively they might be expected to warrant similar correction maneuvers in the coronal plane. However, when viewed in the sagittal plane, the first example is a normokyphotic case whereas the second is a hypoky- photic case. Likewise in Figure 5, the 2 patients have similar kyphosis measures, both are normokyphotic, but 1 is a nonsurgical case while the other is a surgical case. Thus, not all these Lenke- 1 thoracic curves were hypokyphotic. In addition, the location of the apical vertebrae in the scoliotic segment, as usually identified in the coronal plane, may not be the same as that identified in the kyphotic segment when viewed in the sagittal plane. As a result, when the surgeon is contouring the rod based on the apparent deformity in the coronal plane, the resulting correction may not necessarily translate to that of retaining normal kyphosis as one would hope for. It may instead result in flattening or over kyphosing the sagittal curve. This raises the question: if we consider the obvious similarities in the coronal or sagittal planes will the cases undergo the same instrumentation strategies?

In the top views of these 4 cases, the PMC orientations are clearly different. In the nonsurgical case it is closer to the sagittal plane, whereas in the 3 surgical cases it is more rotated. The values of the PMC orientations are very low in the normokyphotic (nonsurgical) cases, higher in the normokyphotic surgical cases (G2), and much higher in the hypokyphotic surgical cases (G3). This suggests that all 4 cases should be treated differently.

Viewing the spine in a perspective other than the anatomic plane projections (sagittal and coronal), like the PMC described in this study, is not entirely a new con- 
Figure 6. The daVinci view representation. The picture on the left shows the coronal and sagittal radiographs, the spline curve representing the spine and the planes of maximum curvature (PMC) for the proximal thoracic (PT), main thoracic (MT), and thoracolumbar/lumbar (TL/L) segments. The true daVinci view is a top view projection of the 3 planes of maximum curvature. The simplified daVinci representation is illustrated on the right wherein the triangles are replaced by an arrow projecting from the center to the apex of the triangle. The length of the arrow is proportional to the curvature, its horizontal projection reflects the magnitude of coronal deformity while its vertical projection reflects sagittal component of the deformity. Position of the CSVL (black dot) with respect to CHVA (origin) is also illustrated in the true daVinci view on the left.

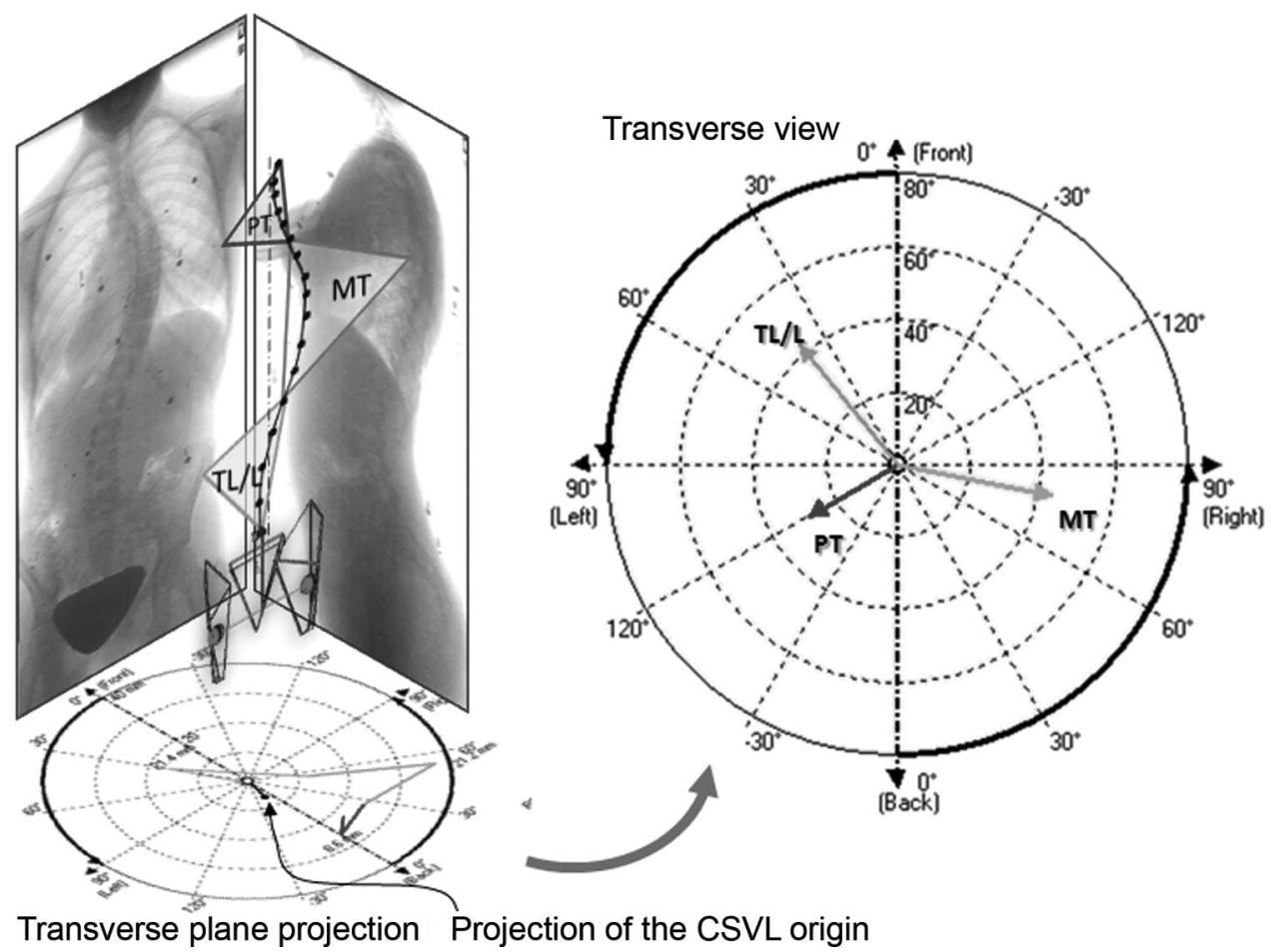

cept. For instance, Stagnara's plan d'élection, ${ }^{28}$ an old concept introduced a long time ago, viewed the spine using an oblique perspective, according to the most rotated vertebra along the local vertebral frontal plane. However, the PMC is a different concept as it is a regional viewing of the spine according to the relative position of the apical vertebra with respect to the end vertebrae of a given spine segment. In a symmetrical spine PMC lies in the sagittal plane. In the presence of scoliosis, its orientation represents a composition of the coronal plane deformity and of the sagittal plane physiologic kyphosis (Figure 6), which may not always correspond to projections in the sagittal and coronal planes that are normally used in surgical planning.

In this analysis as well as in that by Aubin et al and by Villemure et $a l,{ }^{22,2}$ the PMC orientation has been identified as an influential parameter descriptive of the scoliotic deformity. However, it is not a measure that is used in the clinics primarily because of the difficulty in obtaining images of the PMC, or the need for 3D reconstruction software dedicated for evaluating the PMC and in quantifying it.

Careful attention must be given to the interpretation of the perceived spine curvature in the PMC plane. One possible strategy to derive clinically meaningful information from the PMC measure is to examine the spine projections in the transverse view and relate the measure to the more commonly used sagittal and coronal projections to establish its clinical relevance. The SRS committee recently introduced a schematic representation called the daVinci view (see Figure 6). The "daVinci representation" (right panel of Figure 6) is a comprehensive visual representation of the scoliotic spine that illustrates the orientation of the planes of maximum curvature of the 3 spine segments in the transverse view. The center of the circular plot is the point where the central hip vertical axis (CHVA, CHVA is a true vertical bisecting the bi-femoral head axis, often considered to represent the physiological center of balance of the spine-pelvic unit) intersects the transverse plane and every arrow radiating from the center represents a spine segment (PT, MT, and TL/L). The length of the arrow is proportional to the amplitude of the curve, its coronal projection reflects coronal deformity (measured using the Cobb method) while its sagittal projection reflects the sagittal curvature. Thus Cobb angles and sagittal curvature measures of the PT, MT, and TL/L spine segments are reflected in the respective coronal and sagittal components as illustrated in Figure 6 (right panel). The orientation of the arrows relative to the sagittal plane is a measure of the PMC orientation for the different spine segments. The position of the central sacral vertical line (CSVL, CSVL is a true vertical in the coronal plane drawn through the posterior center of the $\mathrm{S} 1$ endplate) relative to the CHVA can also be viewed by their respective projections [CSVL: black dot connected to the origin (CHVA)] in the transverse plane projection of the spine (illustrated in the left panel of Figure 6). The relative orientation of the CSVL and CHVA reflects the pivotal balance between the spine and the pelvis.

If we revisit the cases illustrated in Figures 4 and 5, and examine the respective daVinci views (Figure 7), the nonsurgical example (Figure 7A) has low PMC orientation, moderate PMC values for the 2 examples from $G 2$ (Figure 7B, C) and relative high PMC value in the example from G3 (Figure 7D). The nonsurgical example has a 

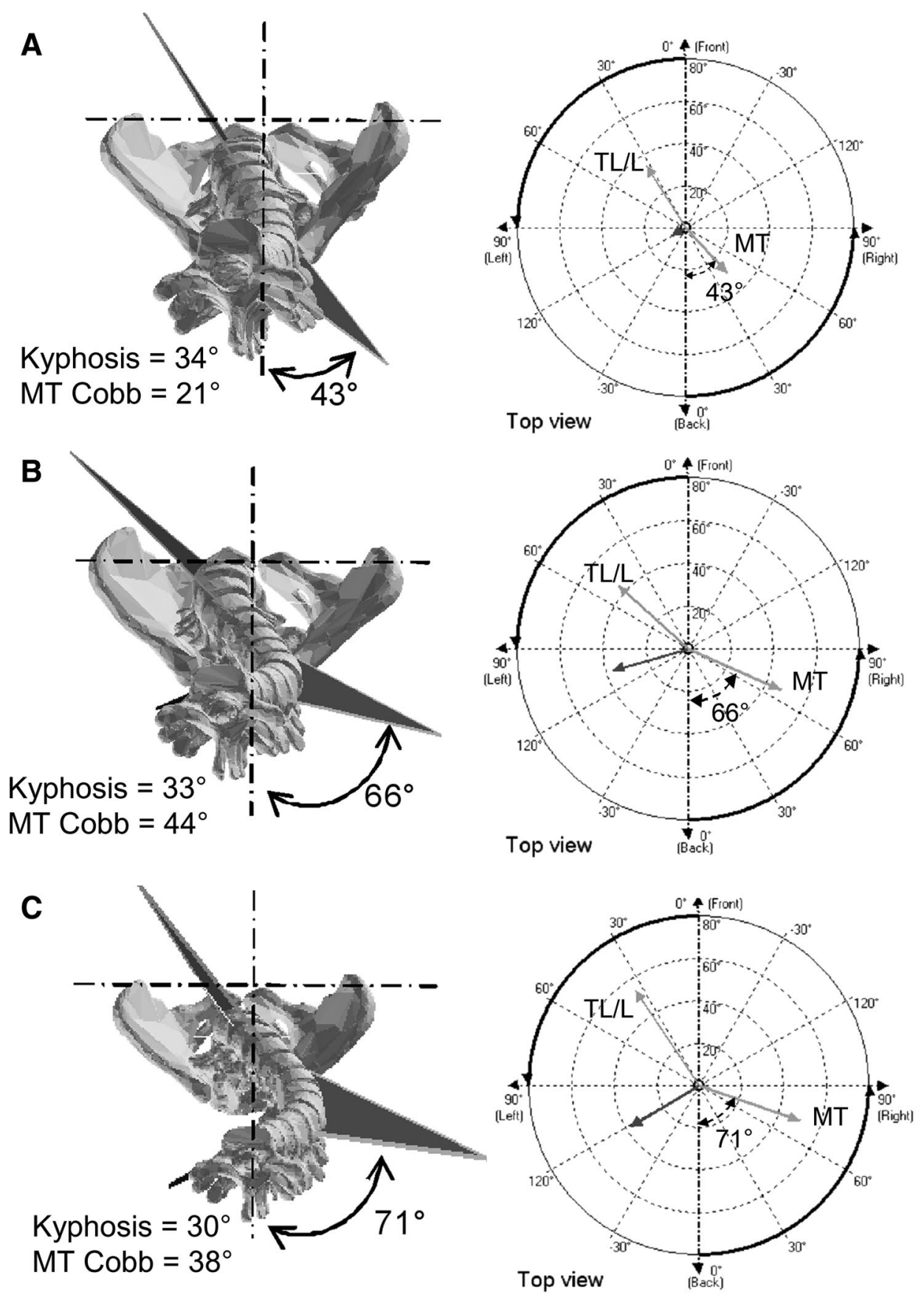

Figure 7. Top views (left column) of the representative cases from Figures 4 and 5 the respective daVinci representations (right column). A, Representative case from G1, B, C, Representative case from G2, D, Representative case from G3. The dotted arrow shows the PMC orientation as indicated in the respective top views in the left column.
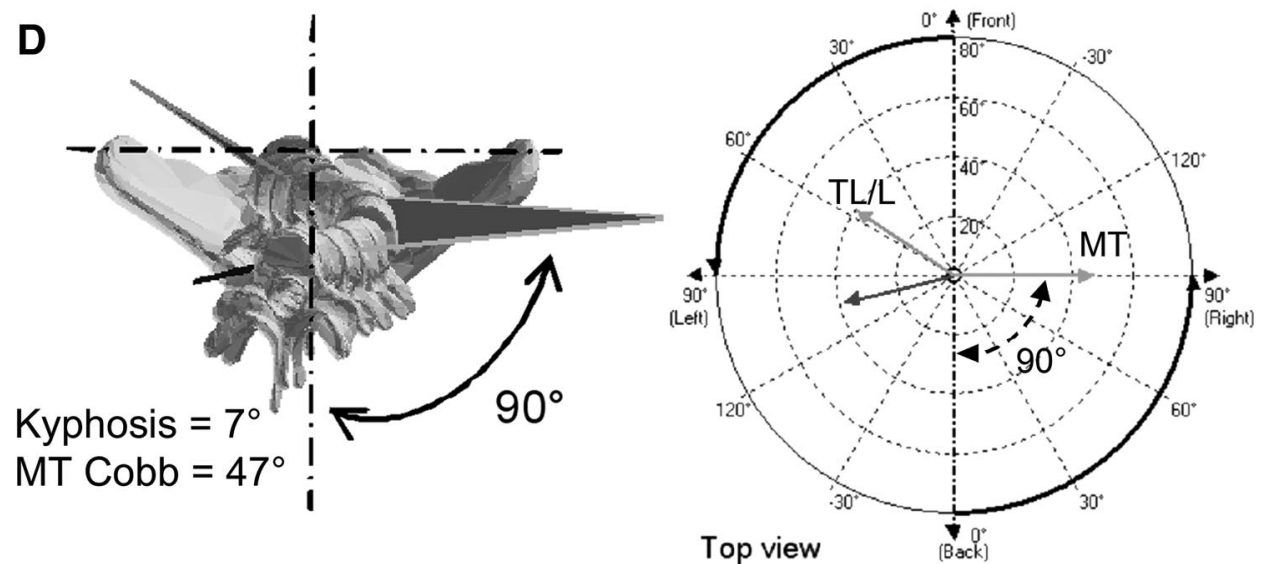
Figure 8. Clinical interpretation of the plane of maximum curvature (PMC) measure. Preoperative radiographs and respective daVinci view (left); Postoperative radiographs and respective daVinci view (right).
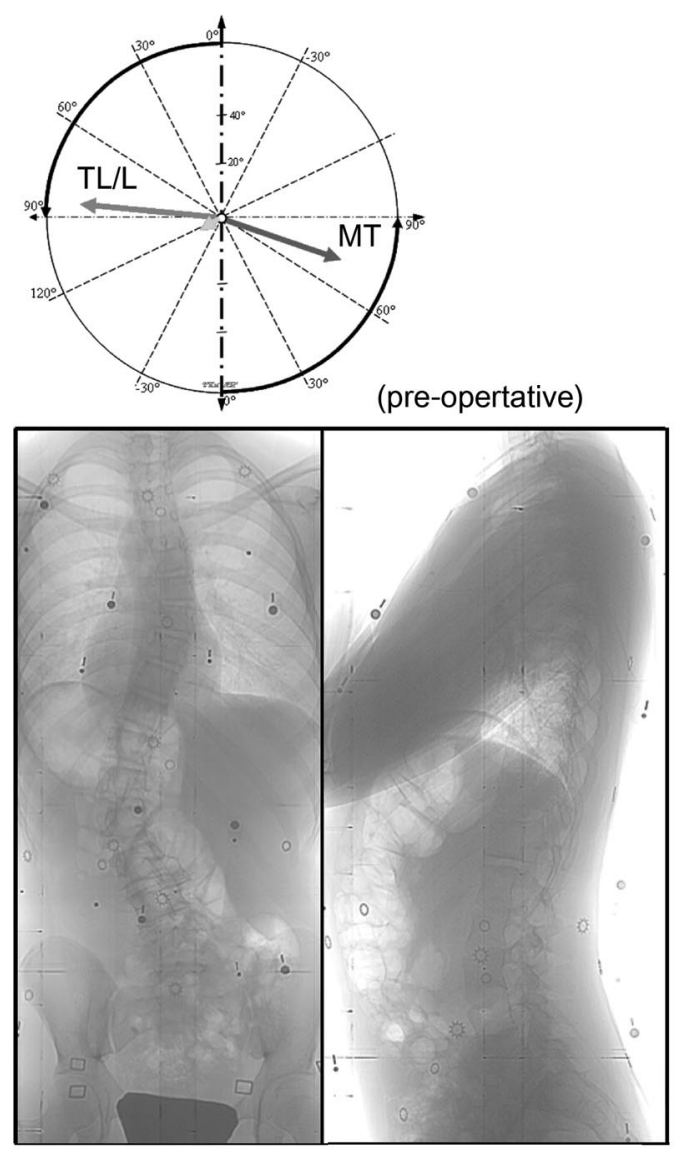
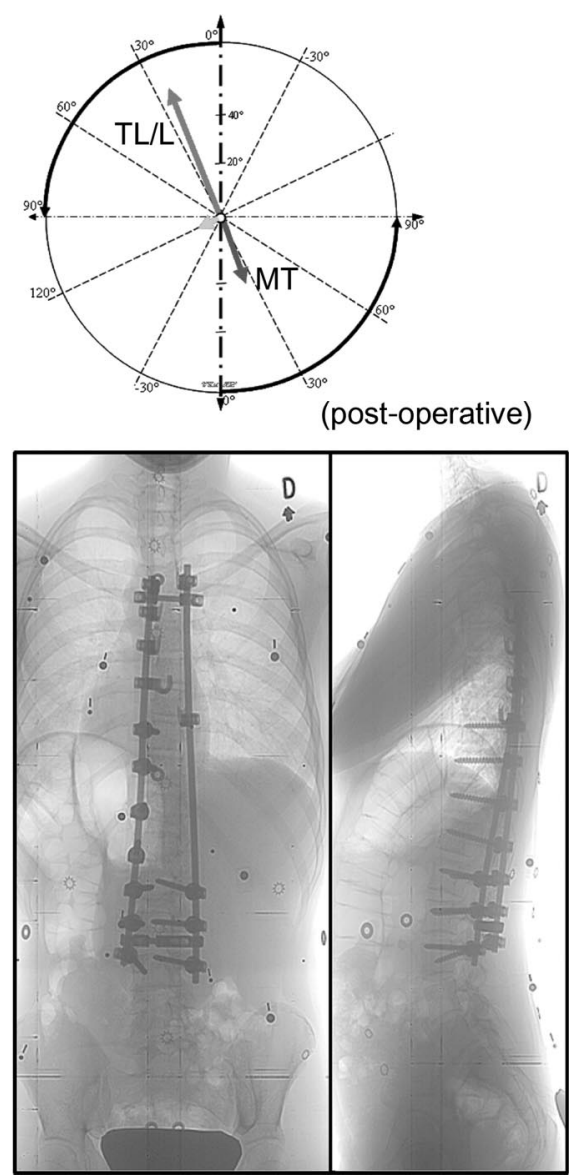

smaller MT Cobb angle, and therefore, a shorter arrow (green) while the remaining 3 examples have almost similar MT Cobb angles which are likewise reflected in the arrow lengths. It is to be noted that the daVinci view also presents the proximal and lumbar characteristics in a similar manner. In this article, however, we have emphasized only the daVinci characterization of the main thoracic parameters.

DaVinci views of preoperative and postoperative spine geometry in a patient are illustrated in Figure 8. As apparent, an optimum surgical correction is that wherein the lumbar curve PMC-arrow is aligned along the vertical directed upwards, whereas the main thoracic curve PMC-arrow is aligned along the vertical directed downwards. The daVinci representation thus provides a very concise and comprehensive visual characterization of the scoliotic deformity and assists in visualizing the correction that a surgeon could possibly try to achieve.

This study involved patients with a single typethoracic structural curves. Further investigation of the PMC measure is necessary to fully explore its potential by considering other types of scoliotic curves. For instance, the location of the apex in the sagittal and coronal planes may not necessarily coincide with that identified in the planes of maximum curvature. This aspect will have to be considered while using the PMC orientation as a measure to plan the surgical procedure. Nonetheless, the study presents evidence that $3 \mathrm{D}$ characterization of the scoliotic curve reveals inherent structural differences that are not apparent in single planar radiographic assessments and further illustrates the influence of the measure on curve classification.

\section{- Key Points}

- Based on 3D metrics, 3 groups, 1 nonsurgical (minor curves) and 2 surgical (major curves), were identified in 172 Lenke type-I curves.

- Among patients with Lenke Type 1 curves of similar Cobb angle there were 2 subgroups having different degree of kyphosis (T4-T12), apparent in distinctly different orientation of PMC.

- Cluster analysis of spinal shape in 3D reveals differences between patients not evident in 2D radiographs.

- A new means that schematically represent the PMC on the transverse plane (the daVinci representation) is clinically helpful to report $3 \mathrm{D}$ spinal deformities.

\section{Acknowledgments}

The authors thank Juliane Meunier who assisted with some preliminary analysis of the data and Phillipe Labelle for the 3D reconstructions and the support of the SRS. The study was part of a project undertaken by the SRS 3D classification committee. 


\section{References}

1. Carpineta L, Labelle H. Evidence of three-dimensional variability in scoliotic curves. Clin Orthop Relat Res 2003;412:139-48.

2. Villemure I, Aubin CE, Grimard G, et al. Progression of vertebral and spinal three-dimensional deformities in adolescent idiopathic scoliosis: a longitudinal study. Spine 2001;26:2244-50.

3. Delorme S, Petit Y, DeGuise JA, et al. Assessment of the 3D reconstruntion and high-resolution geometric modeling of the human skeletal trunk from 2D radiographic images. IEEE Trans Biomed Eng 2003;50:989-98.

4. Cheriet F, Laporte C, Kadoury S, et al. A novel system for the 3D reconstruction of the human spine and rib cage from biplanar X-ray images. IEEE Trans Biomed Eng 2007;54:1356-8.

5. Kadoury S, Cheriet F, Laporte C, et al. A versatile 3D reconstruction system of the spine and pelvis for clinical assessment of spinal deformities. Med Biol Eng Comput 2007;45:591-602.

6. Ponseti IV, Friedman B. Prognosis in idiopathic scoliosis. J Bone Joint Surg 1950;32A:381-95.

7. King HA, Moe JH, Bradford DS, et al. The selection of fusion levels in thoracic idiopathic scoliosis. J Bone Joint Surg 1983;65:1302-13.

8. Stokes IA, Aronsson DD. Identifying sources of variability in scoliosis classification using a rule-based automated algorithm. Spine 2002;27:2801-5.

9. Stokes IA, Aronsson DD. Computer-assisted algorithms improve reliability of King classification and cobb angle measurement of scoliosis. Spine 2006; 31:665-70.

10. Coonrad RW, Murell GAC, Mothey G. A logical coronal pattern classification of 2000 consecutive idiopathic scoliosis cases based on the scoliosis research society-defined apical vertebra. Spine 1998;1380-1391.

11. Lenke LG, Betz RR, Bridwell KH, et al. Intraobserver and interobserver reliability of the classification of thoracic adolescent idiopathic scoliois. J Bone Joint Surg 1998;80A:1097-106.

12. Lenke LG, Betz RR, Harms J, et al. Adolescent idiopathic scoliosis: a new classification to determine extent of spinal arthrodesis. J Bone Joint Surg 2001;83:1169-81.

13. Lenke LG, Betz RR, Clements D, et al. Curve prevelance of a new classification of operative adolescent idiopathic scoliosis. Spine 2002;27:604-11.

14. Puno RM, An KC, Puno RL, et al. Treatment recommendations for idiopathic scoliosis: an assessment of the Lenke classification. Spine 2003;28: 2102-15.

15. Cil A, Pekmezci M, Yazici M, et al. The validity of Lenke criteria for defining structural proximal thoracic curves in patients with adolescent idiopathic scoliosis. Spine 2005;30:2550-5.

16. Cummings RJ, Loveless EA, Campbell J, et al. Interobserver reliability and intraobserver reproducibility of the system of King et al. for the classification of adolescent idiopathic scoliosis. J Bone Joint Surg 1998;80A:1107-11.

17. D'Andrea LP, Betz RR, Lenke LG, et al. Do radiographic parameters correlate with clinical outcomes in adolescent idiopathic scoliosis. Spine 2000;25: 1795-802.

18. Duong L, Cheriet F, Labelle H. Three-dimensional classification of spinal deformities using fuzzy clustering. Spine 2006;31:923-30.

19. Poncet P, Dansereau J, Labelle H. Geometric torsion in idiopathic scoliosis: three-dimensional analysis and proposal for a new classification. Spine 2001; 26:2235-43.

20. Stokes IA. Axial rotation component of thoracic scoliois. J Orthop Res 2005;7:702-8.

21. Robitaille M, Aubin CE, Labelle H. Intra and interobserver variability of preoperative planning for surgical instrumentation in adolescent idiopathic scoliosis. Eur Spine J 2007;16:1604-14.

22. Aubin CE, Lobeau D, Labelle H, et al. Planes of maximum deformity in the scoliotic spine. In: Stokes IAF, ed. Research into Spinal Deformities. Amsterdam, The Netherland: IOS Press; 1999;45-48.

23. Stokes IA, Bigalow LC, Moreland MS. Three-dimensional spinal curvature in idiopathic scoliosis. J Orthop Res 1987;5:102-13.

24. Stokes IA. Three-dimensional terminology of spinal deformity. A report presented to the Scoliosis Research Society by the Scoliosis Research Society Working Group on 3D terminology of spinal deformity. Spine 1994;19: $236-48$.

25. Ball GH, Hall DJ. ISOData - an iterative method of multivariate analysis and pattern classification. Proc IFIPS Congr. 1965.

26. Levinson SE, Rabiner LR, Rosenberg AE, et al. Interactive clustering technique for selecting speaker-independent reference templates for isolated word recognition. IEEE Trans Acoust 1979;27:134-41.

27. Memarsadeghi N, Mount DM, Netanyahu NS, et al. A fast implementation of the ISOData clustering algorithm. In: Proceedings of the IEEE International Geoscience and Remote Sensing Symposium (IGARSS’03); 2003; vol. III:2057-2059; Toulouse, France.

28. du Peloux J, Fauchet R, Faucon B, et al. Le plan d'election pour l'examen radiologique des cyphoscolioses. Rev Chir Orthop Reparatrice Appar Mot 1965;51:517-24. 